\title{
The Effect of $0.01 \%$ Atropine Eye Drops on the Ocular Surface in Children for the Control of Myopia-The Primary Results from a Six-Month Prospective Study
}

This article was published in the following Dove Press journal:

Therapeutics and Clinical Risk Management

\author{
Jingyi Cheng $\mathbb{D}^{1-3, *}$ \\ Yujing Yang ${ }^{1-3, *}$ \\ Xiehe Kong ${ }^{4}$ \\ Li Zeng ${ }^{1-3}$ \\ Zhi Chen $\mathbb{D}^{1-3}$ \\ Jianjiang $X u^{1-3}$ \\ Chaoran Zhang ${ }^{1-3}$
}

'Department of Ophthalmology and Visual Science, Eye, Ear, Nose and Throat Hospital, Shanghai Medical College of Fudan University, Shanghai, People's Republic of China; ${ }^{2}$ Key Laboratory of Myopia, Ministry of Health (Fudan University), Shanghai, People's Republic of China; ${ }^{3}$ Shanghai Key Laboratory of Visual Impairment and Restoration (Fudan University), Shanghai, People's Republic of China; ${ }^{4}$ Shanghai Research Institute of Acupuncture and Meridian, Shanghai, People's Republic of China

*These authors contributed equally to this work

Correspondence: Chaoran Zhang Department of Ophthalmology and Visual Science, Eye, Ear, Nose and Throat Hospital, Shanghai Medical College of Fudan University, 83 Fenyang Road, Shanghai 20003I, People's Republic of China

Tel/Fax +86 2164377/34

Email zhangchaoran@hotmail.com
Purpose: To evaluate the effect of $0.01 \%$ atropine eye drops on the ocular surface in children for the control of myopia.

Methods: A total of 72 participants were recruited for this prospective study. Prior to and after 1,3 , and 6 months of $0.01 \%$ atropine administration, an ocular surface disease index (OSDI) questionnaire was obtained, Keratograph 5M was used for the measurement of the tear meniscus height (TMH), noninvasive keratographic tear film break-up time (NK-BUT, the first keratographic break-up time, $\left[\mathrm{NK}-\mathrm{BUT}_{\text {first }}\right]$ and the average keratographic break-up time, $\left[\mathrm{NK}-\mathrm{BUT}_{\mathrm{ave}}\right]$ ), bulbar redness (BR), meiboscore (MS), and anterior segment optical coherence tomography (AS-OCT) was used to calculate the inferior tear meniscus area (TMA).

Results: After using the $0.01 \%$ atropine eye drops for 1 month, 9 subjects complained of discomfort immediately after administration, but this quickly subsided, and 1 subject was temporarily dazzled. All the ocular surface symptoms were mild and occurred rarely. After 3 months, these complaints no longer occurred. Compared with the baseline values, the OSDI scores $(0.08 \pm 0.28)$, values of TMH $(0.23 \pm 0.04 \mathrm{~mm})$, TMA $\left(0.0420 \pm 0.0444 \mathrm{~mm}^{2}\right)$, NK$\operatorname{BUT}_{\text {first }}(9.39 \pm 5.25 \mathrm{~s}), \mathrm{NK}-\mathrm{BUT}_{\mathrm{ave}}(10.49 \pm 4.94 \mathrm{~s}), \mathrm{BR}(0.63 \pm 0.37)$, and MS $(0.89 \pm 0.70)$ did not change significantly after 6 months of $0.01 \%$ atropine eye drop administration $(\mathrm{P}>0.05)$.

Conclusion: In this 6-month prospective study, no side effects were observed on the ocular surface after using $0.01 \%$ atropine in children.

Keywords: $0.01 \%$ atropine eye drops, ocular surface, children, myopia control

\section{Introduction}

Myopia has emerged as an important cause of correctable visual impairment and preventable blindness in the world, especially in East Asia. ${ }^{1-3}$ Among urban Chinese adolescents, the prevalence of myopia was $78.4 \%$ among the 15 -yearolds in Southern China in 2003, ${ }^{4}$ and $95.5 \%$ among the University students in Shanghai. ${ }^{5}$ It has been estimated that myopia will affect nearly 5 billion people by the year 2050 and become a major public health challenge. ${ }^{6}$

Currently, no effective treatments exist for myopia as its pathogenesis is not fully understood. ${ }^{7}$ Current efforts are focused on how to reduce the progression of myopia. At present, atropine eye drops, orthokeratology, peripheral defocus modifying contact lenses, pirenzepine, and progressive addition lenses for spectacles 
have demonstrated various efficacies at decreasing the rate of axial length extension. ${ }^{8}$ The use of $0.01 \%$ atropine is growing rapidly, and becoming the most common strategy for myopia control in children of Asian countries. ${ }^{9}$ Even though the National Medical Products Administration in China has not formally approved low-concentration atropine eye drops for the control of myopia in children, its use is popular in mainland China via foreign procurement or prepared in the hospitals. ${ }^{10}$

Previous studies have reported some common complications with the topical application of different concentrations of atropine eye drops, such as dilated pupils, photophobia, blurred vision, myopic rebound after drug withdrawal, allergic conjunctivitis, allergy-related dermatitis of the eyelids, and abnormal accommodation. ${ }^{11-14}$ It is worth noting that $1 \%$ atropine eye drops can be used to make dry eye animal models quickly, ${ }^{15}$ and a high incidence $(97.5 \%)$ of dry eye was reported in young children with allergic conjunctivitis in southwest China. ${ }^{16}$ Therefore, when considering long-term use of eye drops in young children, ${ }^{12}$ safety is as important as efficacy, and it is necessary to be cautious about its possible influence on their ocular surfaces. However, to our knowledge, no studies have investigated the effects of atropine eye drops on the ocular surface, especially on the tear film and meibomian gland.

The aim of this prospective study was to evaluate the effect of $0.01 \%$ atropine eye drops on the ocular surface in children, including the tear film and meibomian glands.

\section{Methods}

This prospective study was performed from April 2019 until May 2020 at the Department of Ophthalmology, Eye and ENT hospital, Fudan University, Shanghai, China. This study was performed in accordance with the tenets of the Declaration of Helsinki and was approved by the Medical Ethics Committee of the Eye and ENT Hospital, Fudan University, Shanghai, China. Written informed consent was obtained from all the subjects and their parents after the study was explained to them.

\section{Participants}

Participants were included in the study if they met the following inclusion criteria: 1) aged between 7 and 12 years old; 2) dilated optometry showing spherical equivalent (SE) between $-6.00 \mathrm{DS}$ and $+0.50 \mathrm{DS} ; 3$ ) keratometry 41-46 D; 4) astigmatism $\leq 1.50 \mathrm{DC}$; 5) anisometropia $\leq 1.50 \mathrm{DS} ; 6$ ) intraocular pressure (IOP) between 10 and $21 \mathrm{~mm} \mathrm{Hg}$; 7) no history of atropine use 1 month prior to the study; 8) agreement to be enrolled in the study by both participants and their parents via informed consent.

Participants were excluded if they had: 1) a history of systemic or ocular disease that required treatment, including keratitis, ocular allergic disease, any other ocular surface disease, glaucoma, active or chronic uveitis, or previous ocular surgery or injury; 2) allergy to atropine; 3) history of using atropine eyedrops, orthokeratology, or any method to prevent or control myopia 1 month prior to the study; 4) unreasonable expectations of either participants or their parents.

\section{Ophthalmologic Examination}

After recruitment, the participants underwent a complete ophthalmic examination. All participants were examined by the same experienced examiner, who was blinded for the usage duration, prior to and after 1, 3, and 6 months of $0.01 \%$ atropine administration every night $(0,4 \mathrm{~mL}$ : $0.04 \mathrm{mg}$, SINQI Pharmaceutical Co., Ltd., Shenyang, China). At each visit, the following was done: an ocular surface disease index (OSDI) questionnaire was used for assessing the participants' ocular surface symptoms and severity of dry eye, uncorrected visual acuity measurement (UCVA; E-charts at a distance of $5 \mathrm{~m}$ ), slit-lamp examination (type YZ5E, 66 Vision Tech. Co., China), noncontact IOP tonometry (Full Auto Tonometer TX-F, Canon, Japan), refraction (NIDEK RT-5100), keratometry (WAM-5500), axial length measurement (ZEISS IOL Master), Keratograph 5M Topographer inspection (Oculus Optikgerate GmbH, Wetzlar, Germany), and ASOCT (CASIA SS-1000; Tomey Corporation, Nagoya, Japan; software version 6H.4).

Keratograph $5 \mathrm{M}$ inspection included noninvasive tear meniscus height $(\mathrm{TMH})$, noninvasive keratographic tear film break-up time (NK-BUT, the first keratographic break-up time, [NK-BUT $\left.\mathrm{Nirst}_{\text {f }}\right]$ and the average keratographic break-up time, [NK-BUT $\left.\mathrm{ave}_{\mathrm{a}}\right]$ ), bulbar redness (BR) measurements, and meibography. The degree of meibomian gland dropout, defined as the meiboscore (MS), ${ }^{17}$ was graded as: Grade 0 -no loss of the meibomian gland; Grade $1-$ loss of $<1 / 3$ of the whole gland area; Grade 2 - loss of $1 / 3-2 / 3$ of the whole gland area; and Grade 3 - loss of $>2 / 3$ of the whole gland area. The MS of each eye was calculated as the sum of the scores from both the upper and lower eyelids. 
AS-OCT was used to calculate the inferior tear meniscus area (TMA). The participants were asked to blink before the examination, look straight at a fixation target and maintain spontaneous blinking during the examination. Using the anterior segment three-dimensional scanning pattern, a vertical cross-sectional image of the central cornea could be selected. The eyelid-meniscus junction, the meniscus-eye junction, and the eye-eyelid junction were marked manually. ${ }^{18}$ The programmed calipers measured the value of TMA as the area of the zone surrounded by a closed contour approximated by a spline curve passing through the three points above.

\section{Statistical Analysis}

The statistical analysis was performed on a commercially available statistical software package (SPSS for Mac, version 23.0). Statistically, no significant differences were found between the right and left eyes for any parameters; therefore, only the data from the right eye were used for further statistical analysis.

The categorical variables were expressed as frequencies and constituent ratios. The continuous variables were expressed as means and standard deviations (SD). Statistical comparisons between baseline and different time points were made with the nonparametric Friedman test. A P-value $<0.05$ was defined as statistically significant.

\section{Results}

Seventy-two participants (72 eyes), aged between 7 and 12 were enrolled in this study. The demographic and ophthalmic characteristics of the participants at baseline are shown in Table 1. Among the 72 participants, 1 person left the study after 1 month, and 6 people left after 3 months, because they started to use orthokeratology lenses in addition to $0.01 \%$ atropine eye drops to slow down the progression of myopia.

The ocular surface symptoms of the participants are shown in Table 2. All the symptoms were mild and rarely occurred. The feeling of a dry eye mainly appeared in the morning or after school. After using $0.01 \%$ atropine eye drops for 1 month, 9 subjects (12.5\%) complained of discomfort immediately after administration, including soreness and mild tingling, but this subsided after a few seconds, and 1 subject $(1.39 \%)$ was temporarily dazzled after administration. After 3 months, the participants no longer had these complaints; however, 1 participant did develop a stye.
Table I Demographics and Ophthalmic Characteristics of the Participants

\begin{tabular}{|l|l|l|}
\hline \multicolumn{2}{|l|}{} & Participants \\
\hline No & Male & 72 \\
\hline Sex $(\mathrm{n}, \%)$ & Female & $40(55.56 \%)$ \\
& & $32(44.44 \%)$ \\
\hline Age & $9.49 \pm 1.61$ \\
AL $(\mathrm{mm})$ & $24.38 \pm 0.84$ \\
K (D) & $43.24 \pm 1.37$ \\
ACD (mm) & $3.77 \pm 0.18$ \\
CCT $(\mu \mathrm{m})$ & $547.76 \pm 30.79$ \\
IOP (mmHg) & $15.28 \pm 2.62$ \\
UCVA LOGMAR & $0.40 \pm 0.26$ \\
SE (DS) & $-2.03 \pm 1.21$ \\
\hline
\end{tabular}

Note: The continuous variables were expressed as means and standard deviations (SD).

Abbreviations: AL, axial Length; $K$, keratometry; $A C D$, anterior chamber depth; $\mathrm{CCT}$, central cornea thickness; IOP, intraocular pressure; UCVA, uncorrected visual acuity; SE, spherical equivalent.

Table 2 Symptoms Prior to and After Using 0.01\% Atropine

\begin{tabular}{|l|l|l|l|l|}
\hline & Baseline & I Month & 3 Months & 6 Months \\
\hline No & 72 & 72 & 71 & 65 \\
Dry eye (n, \%) & $4(5.56 \%)$ & $2(2.78 \%)$ & $2(2.82 \%)$ & $1(1.54 \%)$ \\
Itching (n, \%) & $1(1.39 \%)$ & $2(2.78 \%)$ & $2(2.82 \%)$ & $1(1.54 \%)$ \\
Pink eye (n, \%) & $1(1.39 \%)$ & 0 & 0 & 0 \\
Discomfort* $(\mathrm{n}, \%)$ & 0 & $9(12.5 \%)$ & 0 & 0 \\
Dazzled (n, \%) & 0 & $1(1.39 \%)$ & 0 & 0 \\
Stye (n, \%) & 0 & 0 & $1(1.41 \%)$ & 0 \\
\hline
\end{tabular}

Note: *Discomfort including soreness and mild tinglings.

Compared with the baseline values, the OSDI scores $(P=0.089)$, values of TMH $(P=0.294)$ and TMA $(P=0.459)$, NK-BUT $_{\text {first }}(P=0.355)$ and NK-BUT $_{\text {ave }}(P=0.336), B R(P=0.057)$, and MS $(\mathrm{P}=1.000)$ did not change significantly after 6 months of administering $0.01 \%$ atropine drops (Table 3 ).

\section{Discussion}

To our knowledge, this is the first prospective study to evaluate the effect of $0.01 \%$ atropine eye drops on the ocular surface. Our results showed that after 6 months of $0.01 \%$ atropine use, the subjective parameter, ie, the OSDI questionnaire, and the objective parameters including TMH, TMA, NK-BUT, BR, and MS did not change significantly.

The OSDI is a valid and reliable method for evaluating the severity of dry eye disease, even in children. ${ }^{19,20}$ In 
Table 3 Comparisons of the Ocular Surface Parameters Between Baseline and Different Timepoints

\begin{tabular}{|l|l|l|l|l|l|}
\hline & Baseline & I Month & 3 Months & 6 Months \\
\hline No & 72 & 72 & $7 I$ & 65 & P \\
OSDI & $0.08 \pm 0.28$ & $0.11 \pm 0.32$ & $0.06 \pm 0.23$ & $0.02 \pm 0.12$ \\
TMH $(\mathrm{mm})$ & $0.23 \pm 0.04$ & $0.24 \pm 0.04$ & $0.23 \pm 0.04$ & $0.23 \pm 0.04$ & 0.089 \\
TMA $\left(\mathrm{mm}^{2}\right)$ & $0.0420 \pm 0.0444$ & $0.0385 \pm 0.0174$ & $0.0376 \pm 0.0146$ & $0.0381 \pm 0.0145$ \\
NK-BUT $_{\text {first }}(\mathrm{s})$ & $9.39 \pm 5.25$ & $9.66 \pm 4.73$ & $8.61 \pm 4.16$ & $10.22 \pm 3.89$ & 0.294 \\
NK-BUT $_{\text {ave }}(\mathrm{s})$ & $10.49 \pm 4.94$ & $11.04 \pm 4.36$ & $10.10 \pm 3.50$ & $11.12 \pm 3.20$ & 0.459 \\
BR & $0.63 \pm 0.37$ & $0.53 \pm 0.27$ & $0.55 \pm 0.34$ & $0.49 \pm 0.27$ & 0.335 \\
MS & $0.89 \pm 0.70$ & $0.89 \pm 0.70$ & $0.88 \pm 0.70$ & $0.85 \pm 0.62$ & 0.057 \\
\hline
\end{tabular}

Notes: The continuous variables were expressed as means and standard deviations (SD). Statistical comparisons between baseline and different time points were made with nonparametric Friedman test.

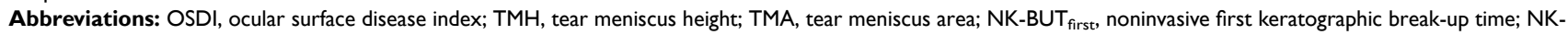
$\mathrm{BUT}_{\text {ave, }}$, noninvasive average keratographic break-up time; $\mathrm{BR}$, bulbar redness; $\mathrm{MS}$, meiboscore.

addition, the noninvasive Keratograph $5 \mathrm{M}$ and AS-OCT were used in the present study, so as to overcome any coordination difficulties in the young participants and to make the results more precise, considering the tear volume, evaporation and meibomian gland.

Atropine mainly acts on M-type cholinergic receptors, leading to increased levels of acetylcholine at the $\beta 2$ receptor level, ${ }^{21}$ which reduces tonic accommodation of the ciliary muscle and effectively decreases initial near work-induced transient myopia. Atropine at concentrations higher than $0.01 \%$ has also been shown to slow down myopic progression, but the clinical use of higher doses of atropine may be limited due to side effects such as vision-related glare, photophobia, and blurred vision. ${ }^{11-14}$ The safety of atropine has been studied, but only according to the presenting symptoms. Allergic conjunctivitis and dermatitis were the most common adverse effects in the $0.10 .5 \%$ atropine groups, but these effects were no longer prevalent in the $0.01 \%$ atropine group at a 2 -year follow up. ${ }^{13}$ When low-concentration atropine was used, including $0.05 \%, 0.025 \%$, and $0.01 \%$, vision-related quality of life was similar to those receiving placebo, and no adverse events related to the atropine use were observed. ${ }^{22}$ In another study, no serious complications were noted compared with the placebo group after using $0.01 \%$ atropine for 14 days in a study of the Chinese population. ${ }^{23}$ In accordance with previous studies, no allergy was observed, and our results showed that after 1 month of atropine administration, nine subjects (12.5\%) complained of discomfort, mainly soreness and mild tingling, immediately after administration, but this subsided after a few seconds, and 1 subject (1.39\%) was dazzled, although these symptoms were mild. However, these symptoms no longer occurred when followed up at 3 and 6 months.
Atropine's action is related to pharmacological antagonism of the muscarinic receptors in the lacrimal gland, which causes a significant decrease in aqueous production, with an evident modification of tear stability. It has been described that the ocular surface (cornea, conjunctiva, and accessory lacrimal glands), meibomian glands, and the main lacrimal gland are interconnected by neural reflex loops that maintain an integrated "functional unit." 24 The neural reflex loops are involved in maintaining the normal tear physiology and can be blocked by atropine. ${ }^{25}$ Multiple topical administrations of $1 \%$ atropine significantly reduced the tear volume after 24 hours in an animal model. ${ }^{26}$ Our results showed that after administration of $0.01 \%$ atropine for 6 months, there were no significant changes in the indices for aqueous production and tear stability. This might be related to the low concentration of atropine in the eye drops, and because the eye drops were only used once at night. In addition, we only observed the participants for 6 months, and it is possible that those parameters would change over a longer duration. Further studies are needed to examine the effects of $0.01 \%$ atropine eye drops over a longer time course.

Our study had a few limitations. First, our study used $0.01 \%$ atropine eye drops, although other concentrations such as $0.5 \%, 0.1 \%, 0.05 \%$, and $0.025 \%$ are also used clinically. Further studies on these atropine concentrations are required to determine what effect they may have on the ocular surface after long-term usage. Second, we observed the participants for 6 months, while $0.01 \%$ of atropine eye drops might be administered for $2-5$ years. A longer study period is required to evaluate the ocular surface safety of $0.01 \%$ atropine over this time frame. 


\section{Conclusions}

The results from this 6-month prospective study show that the use of $0.01 \%$ atropine in children causes no effects on the ocular surface that could increase the risk of dry eye. Further studies investigating different atropine concentrations administered for longer periods of time are required to provide scientific promotion for the usage of lowconcentration atropine eye drops, and prevention and control of myopia effectively and safely.

\section{Abbreviations}

OSDI, ocular surface disease index; $\mathrm{TMH}$, tear meniscus height; TMA, tear meniscus area; NK-BUT, noninvasive keratographic tear film break-up time; BR, bulbar redness; MS, meiboscore; AS-OCT, anterior segment optical coherence tomography; SD, standard deviations; IOP, intraocular pressure; AL, axial Length; K, keratometry; ACD, anterior chamber depth; CCT, central cornea thickness; UCVA, uncorrected visual acuity; SE, spherical equivalent.

\section{Funding}

This research was supported by Shanghai Committee of Science and Technology Foundation (Grant No.194419 01000). The sponsor and funding organization had no role in the design or conduct of this research.

\section{Disclosure}

The authors report no conflicts of interest for this work.

\section{References}

1. Morgan IGO-MK, Saw SM. Myopia. Lancet. 2012;379 (9827):1739-1748. doi:10.1016/S0140-6736(12)60272-4

2. Wong YLSS. Epidemiology of pathologic myopia in Asia and worldwide. Asia Pac J Ophthalmol. 2016;5(6):394-402. doi:10.1097/ APO.0000000000000234

3. Morgan IGHM, Rose KA. Epidemic of pathologic myopia: what can laboratory studies and epidemiology tell us? Retina. 2017;37 (5):989-997. doi:10.1097/IAE.0000000000001272

4. He MZJ, Liu Y, Xu J, Pokharel GP, Ellwein LB. Refractive error and visual impairment in urban children in southern China. Invest Ophthalmol Vis Sci. 2004;45:793-799. doi:10.1167/iovs.03-1051

5. Sun J, Zhou J, Zhao P, et al. High prevalence of myopia and high myopia in 5060 Chinese University Students in Shanghai. Invest Ophthalmol Vis Sci. 2012;53(12):7504-7509. doi:10.1167/iovs.118343

6. Holden BAFT, Wilson DA, Jong M, et al. Global prevalence of myopia and high myopia and temporal trends from 2000 through 2050. Ophthalmology. 2016;123(5):1036-1042. doi:10.1016/j.ophtha. 2016.01.006

7. Matsuda KPK. Recent trend of increasing myopia can be traced to infancy. Med Hypotheses. 2019;128:78-82. doi:10.1016/j.mehy. 2019.05.017
8. Huang JWD, Wang Q, et al. Efficacy comparison of 16 interventions for myopia control in children a network meta-analysis. Ophthalmology. 2016;123(4):697-708. doi:10.1016/j.ophtha.2015.11.010

9. The Impact of Myopia and High Myopia. Report of the joint world health organization-brien holden vision institute global scientific meeting on myopia, University of New South Wales, Sydney, Australia. 16-18 March 2015. Geneva: World Health Organization;2017. Licence: CC BY-NC-SA 3.0 IGO

10. Zhao F, Ma JX. Will the long-term use of atropine eye drops in children increase the risk of dry eye? Med Hypotheses. 2019;132:109331. doi:10.1016/j.mehy.2019.109331

11. Shih KCCT, Ng AL, et al. Use of atropine for prevention of childhood myopia progression in clinical practice. Eye Contact Lens. 2016;42(1):16-23. doi:10.1097/ICL.0000000000000189

12. Chia ALQ, Tan D. Five-year clinical trial on atropine for the treatment of myopia 2: myopia control with atropine $0.01 \%$ eyedrops. Ophthalmology. 2016;123(2):391-399. doi:10.1016/j. ophtha.2015.07.004

13. Chia ACW, Cheung YB, et al. Atropine for the treatment of childhood myopia: safety and efficacy of $0.5 \%, 0.1 \%$, and $0.01 \%$ doses. Ophthalmology. 2012;119(2):347-354. doi:10.1016/j.ophtha.2011. 07.031

14. Chia ACW, Wen L, Fong A, Goon YY, Tan D. Atropine for the treatment of childhood myopia: changes after stopping atropine 0.01\%, 0.1\% and 0.5\%. Am J Ophthalmol. 2014;157(2):451-457. e451. doi:10.1016/j.ajo.2013.09.020

15. Burgalassi SPL, Chetoni P, Saettone MF, Boldrini E. Development of a simple dry eye model in the albino rabbit and evaluation of some tear substitutes. Ophthalmic Res. 1999;31(3):229-235. doi:10.1159/ 000055537

16. Chen L, Pi L, Fang J, Chen X, Ke N, Liu Q. High incidence of dry eye in young children with allergic conjunctivitis in Southwest China. Acta Ophthalmol. 2016;94:e727e730. doi:10.1111/aos.13093

17. Arita KI R, Maeda S, et al. Proposed diagnostic criteria for obstructive meibomian gland dysfunction. Ophthalmology. 2009;116 (11):2058e2051-2063e2051. doi:10.1016/j.ophtha.2009.04.037

18. Bartuzel MMS-ID, Iskander DR. Automatic dynamic tear meniscus measurement in optical coherence tomography. Biomed Opt Express. 2014;5:2759-2768. doi:10.1364/BOE.5.002759

19. Schiffman RM, Jacobsen MDC,G, Hirsch JD, Reis, BL. Reliability and validity of the ocular surface disease index. Arch Ophthalmol. 2000;118(5):615-621. doi:10.1001/archopht.118.5.615

20. Gunay M. GC, E. Yildiz et al. Ocular surface characteristics in diabetic children. Curr Eye Res. 2016;41(12):1526-1531. doi:10.3109/ 02713683.2015.1136421

21. Vasudevan B, Ciuffreda KJ, Ludlam DP. Accommodative training to reduce nearwork-induced transient myopia. Optom Vis Sci. 2009;86:1287-1294. doi:10.1097/OPX.0b013e3181bb44cf

22. Yam JC, Jiang Y, Tang SM, et al. Low-concentration atropine for myopia progression (LAMP) study. Ophthalmology. 2019;126 (1):113-124. doi:10.1016/j.ophtha.2018.05.029

23. Guo L, Fan L, Tao J, et al. Use of topical $0.01 \%$ atropine for controlling near work-induced transient myopia: a randomized, double-masked, placebo-controlled study. J Ocul Pharmacol Ther. 2020;36(2):97-101. doi:10.1089/jop.2019.0062

24. Stern ME, Beuerman RW, Fox RI, Gao J, Mircheff AK, Pflugfelder SC. The pathology of dry eye: the interaction between the ocular surface and lacrimal glands. Cornea. 1998;17:584-589. doi:10.1097/00003226-199811000-00002

25. Schoenwald RD, Vidvauns S, Wurster DE, Barfknecht CF. The influence of tear proteins on the film stability of rabbit tear extracts. J Ocul Pharmacol Ther. 1998;14:15-29. doi:10.1089/jop.1998.14.15

26. Bucolo C, Musumeci M, Salomone S, et al. Effects of topical fucosyl-lactose, a milk oligosaccharide, on dry eye model: an example of nutraceutical candidate. Front Pharmacol. 2015;6:280. doi:10.3389/fphar.2015.00280 


\section{Publish your work in this journal}

Therapeutics and Clinical Risk Management is an international, peerreviewed journal of clinical therapeutics and risk management, focusing on concise rapid reporting of clinical studies in all therapeutic areas, outcomes, safety, and programs for the effective, safe, and sustained use of medicines. This journal is indexed on PubMed Central, CAS,
EMBase, Scopus and the Elsevier Bibliographic databases. The manuscript management system is completely online and includes a very quick and fair peer-review system, which is all easy to use. Visit http://www.dovepress.com/testimonials.php to read real quotes from published authors. 